\section{A CLÍNICA DA EDUCAÇÃO FÍSICA NAS POLÍTICAS PÚBLICAS DE SAÚDE: INTERFACES ENTRE NÚCLEO E CAMPO}

THE PHYSICAL EDUCATION CLINIC IN THE HEALTH PUBLIC POLICES: INTERFACES BETWEEN NUCLEUS AND FIELD C P

LA CLÍNICA DE LA EDUCACIÓN FÍSICA EN LAS POLIITICAS PÚBLICAS DE SALUD: INTERFACES ENTRE NÚCLEO Y CAMPO CP

doi' https://doi.org/10.22456/1982-8918.116321

Luiz Alberto dos Santos Ferreira*<luizrecre@yahoo.com.br>

Tonantzin Ribeiro Gonçalves* <tonantzinrg@unisinos.br>

Leonardo Trápaga Abi** <leoabib@gmail.com>

*Universidade do Vale do Rio dos Sinos (Unisinos). São Leopoldo, RS, Brasil.

**Universidade do Estado de Minas Gerais (UEMG). Passos, MG, Brasil.
Resumo: Este estudo buscou discutir a inserção de Profissionais de Educação Física (PEFS) em Políticas Públicas de Saúde (PPS) e analisar as relações entre núcleo e campo. Para a produção de dados foram realizadas visitas ao Núcleo de Apoio à Saúde da Família (NASF), Programa Academia da Saúde (PAS) e equipe de Práticas Integrativas Complementares em Saúde (PICs). Também realizamos entrevistas em profundidade, observações e anotações em diário de campo, empregando-se Análise Temática do material empírico. O estudo apontou que existem similaridades e diferenças nas práticas dos profissionais atuando nas PPS que transcendem o núcleo da Educação Física, sendo que buscam sempre uma maior aproximação com o campo da Saúde. Os achados revelam o potencial da construção de uma Clínica da Educação Física que esteja conectada com as necessidades de saúde da população usuária.

Palavras chave: Educação Física. Saúde Pública. Atenção à Saúde.
Recebido em: 29 jun. 2021 Aprovado em: 09 dez. 2021 Publicado em: 09 fev. 2022

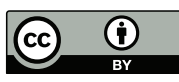

Este é um artigo publicado sob a licença Creative Commons Atribuição 4.0 Internacional (CC BY 4.0). 


\section{INTRODUÇÃO}

A Educação Física (EF) como área do conhecimento e de prática apresenta diversas subáreas de atuação e especialidades, tais como educação, esporte, lazer, saúde, dentre outras. As subáreas da EF também se dividem como no esporte, por exemplo, em que os profissionais atuam no alto rendimento, na escola, no lazer ou na área social. Tais distinções têm requerido dos estudiosos da EF um minucioso cuidado com a caracterização destas subáreas que, por vezes, caminham para a dificuldade de diálogo entre elas. Hallal e Melo (2017) destacam que a falta de diálogo entre as subáreas da EF pode, inclusive, dificultar a criação de novos campos de atuação e a inserção de profissionais no mundo do trabalho, pois fragmenta o conhecimento e limita o debate entre os pares e com outras áreas do conhecimento.

Ao analisar a inserção de profissionais de EF na subárea da Saúde, Nogueira e Bosi (2017) discutem a tradição militarista de educação dos corpos sob o viés nacionalista, disciplinador e biomédico, tendo como finalidade o desenvolvimento da aptidão física e a segurança nacional, pautada em princípios eugênicos. Tal tendência foi reforçada no Brasil na segunda metade do século XX pelo golpe civil-militar de 1964, que impediu discussões críticas no interior da EF. As autoras ainda pontuam que somente nas décadas de 1970 e 1980, com os movimentos de redemocratização do país, se iniciam aproximações da EF com o campo então denominado Saúde Coletiva ${ }^{1}$. Isso se deu, inicialmente, pela via da Epidemiologia, vertente filiada ao paradigma biologicista e biomédico, sendo que aos poucos a EF passou a se vincular aos estudos das Ciências Humanas e Sociais e, por último, na área das Políticas Públicas de Saúde (PPS) (NOGUEIRA; BOSI, 2017).

A inserção de Profissionais de Educação Física (PEFS) nas PPS é tímida ao longo da história, apesar de sabermos de experiências historicamente mais consolidadas na Política de Saúde Mental. Por exemplo, no Hospital Psiquiátrico São Pedro, localizado em Porto Alegre/RS, sabe-se que a inclusão de PEFS no Serviço de Recreação (atual Serviço de Educação Física) iniciou já em meados dos anos 1970 (SANTOS, 2016). Damico (2007) também relata experiências da década de 1990 com sua inserção na Residência Multiprofissional em Saúde (RMS), formação de pós-graduação que mescla ensino e trabalho nos equipamentos de saúde.

Com a revisão da Portaria MS/GM 154/2008, que criou os Núcleos de Apoio à Saúde da Família (NASF), a área da EF passou a ser contemplada como uma das profissões prioritárias no texto de uma PPS. Dentre os pontos que a Portaria MS/GM 256/2013 (BRASIL, 2013a) trouxe está também a criação do código provisório da Classificação Brasileira de Ocupações "Profissional de Educação Física na Saúde" (2241-E1), fato que passava a permitir que esses profissionais realizassem registros formais de produtividade e tivessem maior acesso a vínculo empregatício². Embora a possibilidade da presença de PEFS na saúde pública já tivesse sido mencionada em documentos anteriores, é com a reedição da portaria do NASF, em 2013, que se abre

\footnotetext{
1 De acordo com Paim e Almeida-Filho (1998), podemos entender a Saúde Coletiva enquanto um campo de práticas e saberes interdisciplinar em que se busca a produção de conhecimentos a respeito do fenômeno saúde-doença como processo social e histórico, bem como sobre o trabalho e as políticas públicas em saúde. 
espaço para uma inserção mais efetiva destes profissionais nas PPS. Na sequência, vieram as normativas que incluíram esses profissionais em outros dispositivos, como o Programa Academia da Saúde - PAS (BRASIL, 2013b)3 e, por último, na criação da nova modalidade de Centros de Atenção Psicossocial (CAPS) de tipo IV (BRASIL, 2017a).

A produção acadêmica da EF em relação à inserção nas PPS reflete uma dualidade conceitual quanto a definição da práxis. O conceito de práxis acompanha a humanidade desde os pensadores da Grécia antiga, como Platão, que trabalhou a dicotomia entre o mundo inteligível e sensível. As formulações de Hegel rompem com essa dualidade, igualando a práxis material ao trabalho espiritual que conduz ao espírito absoluto, em que mesmo o homem escravizado, na medida em que toma consciência do trabalho material, liberta-se justamente por esta produção, condenando seu senhor, pela dependência da sua produção (MACHADO, 2018). Aqui, entendemos práxis no sentido marxista do conceito (MARX; ENGLES, 2007) para nos referirmos à impossibilidade de dissociar teoria e prática e ao sentido da ação transformadora, essencialmente humana, que promove compreensão da realidade e consciência na ação (SILVA, 2017).

No campo da Saúde, o entendimento da práxis da EF oscila entre o paradigma biologicista, centrado em estudos e práticas que remetem a questões anatômicas, cinesiológicas e bioquímicas, e os estudos socioculturais, que levam em consideração aspectos subjetivos e históricos do desenvolvimento individual e coletivo dos povos e territórios (LUZ, 2007). Esses dissensos levam a disputas que, por vezes, não fazem sentido no dia a dia dos profissionais, como sugerem Damico e Knuth (2014) ao discutirem os usos e apropriações das noções de Práticas Corporais e Atividade Física.

As práticas corporais estariam ligadas às questões subjetivas e socioculturais, enquanto a atividade física se relacionaria a elementos biológicos, demarcando "um enfrentamento epistemológico inoperante no cotidiano dos serviços de saúde" (DAMICO; KNUTH, 2014, p. 329). Tais disputas têm trazido dificuldades de compreensão da práxis cotidiana dos serviços de saúde, ao criarem uma crença na necessidade de filiação a uma ou outra vertente. Nesse sentido, Bilibio e Damico (2011) defendem um movimento crítico para pensar a práxis dos PEFS, pautado por uma atitude ética e estética questionadora e aberta a diversas possibilidades e linguagens de vida e intervenção.

Pesquisas anteriores (FERREIRA et al., 2017; FURTADO et al., 2016) debateram aspectos da práxis de PEFS em serviços de Saúde Mental, discutindo a necessidade de uma inserção efetiva nas equipes multiprofissionais de Saúde e não como mero realizador de tarefas destinadas aos saberes e práticas do núcleo da EF. Nesses trabalhos, tomou-se como base o conceito de campo e núcleo (CAMPOS, 2000), em que o campo é definido como um espaço sem limites definidos ou demarcados para a atuação, enquanto núcleo define um determinado saber ou práxis profissionais. A partir dessas definições, se buscará ampliar as reflexões em torno da inserção do PEFS em outras PPS, como o PAS, Núcleo Ampliado de Saúde

3 A Portaria MS/GM 719/2011, que institui o Programa Academia da Saúde no âmbito do SUS, foi revisada e revogada após a publicação da Portaria MS/GM 2681/2013. 
da Família e Atenção Básica (NASF-AB) e a Política Nacional de Práticas Integrativas e Complementares (PNPIC).

Por ser a Saúde Pública ${ }^{4}$ um campo de prática emergente dos PEFS e envolto em debates acadêmicos, não surpreende que a pesquisa empírica sobre o tema seja também incipiente. Nesse sentido, Vaz (2014) realizou um estudo sobre a distribuição de PEFS no estado do Rio Grande do Sul por meio de uma busca no Cadastro Nacional de Estabelecimentos de Saúde (CNES). Verificou-se que dos 497 municípios do estado apenas 245 possuíam PEFS, os quais se inseriam em diferentes PPS (Atenção Básica, Saúde Mental, Policlínicas, Clínicas Especializadas, Hospitais Gerais e Gestão da Saúde). A maioria dos profissionais, 79\%, estava vinculada à rede pública e $21 \%$ à rede complementar. Em que pese a baixa qualidade das informações registradas no CNES, o levantamento revelou que a maior parte destes profissionais estava concentrada na Política Nacional de Atenção Básica (PNAB), devido ao maior desenvolvimento de políticas como NASF e PAS (VAZ, 2014).

Assim, considerando o crescente aumento da inserção dos PEFS nas PPS e a necessidade de problematizar a formação na área e a articulação com os diferentes campos de práticas abertos no SUS, este estudo se propõe a analisar a inserção de PEFS em equipes de PAS, NASF-AB e de Práticas Integrativas e Complementares em Saúde de municípios da Região Metropolitana de Porto Alegre, seus desafios e possibilidades. Ainda, a partir do trabalho de campo, se busca refletir sobre a construção de uma possível clínica da EF no campo da Saúde.

\section{METODOLOGIA}

O estudo se caracteriza por uma pesquisa qualitativa que se circunscreve ao campo da inserção de PEFS nas PPS da Região Metropolitana de Porto Alegre/RS. A abordagem qualitativa responde a questões muito particulares, preocupando-se com as ciências sociais no nível da realidade. Sendo assim, trabalha com atitudes, crenças, aspirações e motivos, se baseia na qualidade e não na quantidade (CAMPOS et al., 2012). Para Turato (2003), a pesquisa qualitativa não tenciona generalizar resultados, mas foca sua atenção no específico, no peculiar, no individual, buscando a compreensão e não a explicação do fenômeno estudado.

O trabalho de campo se deu durante 12 meses de inserção no PAS, Práticas Integrativas e Complementares em Saúde e NASF-AB ${ }^{5}$ em três cidades da Região Metropolitana de Porto Alegre, com imersões nos serviços, conversas (virtuais e presenciais) e entrevistas com gestores e usuários. Também foram realizadas visitas periódicas aos equipamentos das PPS (pelo menos 24 visitas antes da pandemia), entrevistas com os PEFS.

As observações dos processos de trabalho foram registradas em diário de campo. O recorte analítico do presente manuscrito enfoca as observações e entrevistas com os PEFS e uma gestora.

4 Ao utilizar o termo "Saúde Pública" estamos nos referindo especificamente aos serviços públicos de saúde (que fazem parte da rede do SUS) em que os PEFS estão se inserindo formalmente.

5 De acordo com a nova versão da Política Nacional de Atenção Básica (BRASIL, 2017b), instituída pela Portaria MS/GM 2436/2017, o Núcleo de Apoio à Saúde da Família passa a ser chamado de Núcleo Ampliado de Saúde da Família e Atenção Básica (NASF-AB). 
A pesquisa teve a aprovação da Secretaria da Saúde do Estado do Rio Grande do Sul (SES/RS) e dos Núcleos de Educação e Saúde Coletiva (NUMESC) dos municípios de Esteio e Sapucaia do Sul, além do Comitê de Ética da instituição proponente do estudo. Todos os colaboradores e colaboradoras da pesquisa assinaram um Termo de Consentimento Livre e Esclarecido (TLCE).

Os contatos com os colaboradores e colaboradoras foram feitos de forma presencial a partir de novembro de 2019 e, após março de 2020, à distância, em função da pandemia de covid-19. As entrevistas foram realizadas individualmente, utilizando roteiro semiestruturado - o qual foi conduzido de modo semidirigido -, a fim de permitir maior flexibilidade e preservar a construção narrativa dos(as) colaboradores(as). Os roteiros elaborados para PEFS e Gestores abordaram temáticas relacionadas à formação, trajetória profissional, rotina de trabalho, percepção sobre atuação nas PPS e inserção da EF. Foram realizadas entrevistas com seis PEFS (dois homens e quatro mulheres), representando a totalidade dos que atuavam nas três PPS pesquisadas. Também foi entrevistada a coordenadora do PAS. O estudo planejava ainda entrevistar oito usuários, contudo, em virtude da interrupção das atividades por causa da pandemia, não foi possível entrevistá-los. Para citar as contribuições dos participantes durante o texto, utilizaremos a letra "P" seguida dos números 1 a 6 para os PEFS e C7 para designar a coordenadora.

Para análise e discussão dos achados empíricos foi realizada uma Análise Temática (BRAUN; CLARKE, 2006; SOUZA, 2019). Esta técnica busca identificar, analisar, interpretar e relatar padrões (temas) a partir da empiria e produção dos dados qualitativos. A Análise Temática organiza e descreve o conjunto de dados em detalhes, organizando-se em seis fases. A primeira é a familiarização com os dados; na segunda se geram dados e códigos iniciais; na terceira descrevem-se temas iniciais; na quarta se revisam os temas; depois se definem e nomeiam os temas definitivos; e, por fim, se produz o relato da análise na articulação entre os dados, a pergunta da pesquisa e a literatura (SOUZA, 2019).

\section{RESULTADOS E DISCUSSÃO}

A seguir, serão apresentados e discutidos os temas gerados a partir da análise do material empírico, ilustrando-os com vinhetas das entrevistas e do diário de campo. O movimento analítico gerou três categorias temáticas, intituladas: "O Núcleo da Educação Física na interface com o Campo da Saúde: fragilidades na formação"; "Trabalho em Equipe e dia a dia das PPS: junto e misturado" e "Clínica da Educação Física: uma clínica mutante”.

\subsection{O NÚCLEO DA EDUCAÇÃO FÍSICA NA INTERFACE COM O CAMPO DA SAÚDE: FRAGILIDADES NA FORMAÇÃO}

Nas três políticas pesquisadas percebemos relações diferentes entre o campo da Saúde e o núcleo da EF, sendo que os PEFS tinham diferentes processos de trabalho. Nas atividades relacionadas às PICs, os PEFS eram demandados a ter uma formação específica para o tipo de intervenção que fosse ser executada (por 
exemplo: para dar aulas de loga seria preciso ter também algum tipo de formação em loga). Desta forma, ter ou não formação inicial em EF não era fundamental para a inserção de PEFS na política. Nas PICs, o PEFS colaborador da pesquisa era gestor da política no RS e nos falou dos debates sobre a normatização desta atuação:

Hoje atuando como trabalhador, na gestão de uma política, o conhecimento da Educação Física fica em segundo plano, não dando subsídio para isso que eu faço. [...] Estamos falando de uma outra racionalidade, os conteúdos que a gente não tem nas matrizes curriculares, então não basta tu ser graduado em Educação Física para conseguir ofertar o Tai Chi Chuan. Então, nesse momento é melhor que a pessoa tenha uma expertise em Tai Chi Chuan que a graduação em Educação Física. (P1, entrevista).

Diferentemente das PICs, no NASF-AB há um equilíbrio, dando outro sentido à inserção da EF para o trabalho na política. Os processos de trabalho dos PEFS incluíam tanto práticas circunscritas ao núcleo (como grupos de ginástica, atendimento personalizado, práticas de reabilitação física, atividades de lazer, entre outras), quanto práticas do campo (como matriciamento, reuniões de equipe e atendimentos individuais):

Minha semana no NASF é bastante diversificada, por exemplo, há turnos para planejamento, grupos de educação em saúde, grupos de convivência, turnos de atendimento individual, matriciamento, reunião de saúde mental no hospital, reunião de equipe. (P2, notas de diário de campo).

Num crescente protagonismo da EF na inserção nas três políticas pesquisadas, no PAS percebeu-se que as atividades dos PEFS eram majoritariamente relacionadas aos saberes do núcleo. Apesar disso, ocorria interação com os demais Núcleos da Saúde e outras PPS, além da interlocução com a rede intersetorial (ou seja, políticas de outras áreas, como cultura, educação e assistência social). A gestora do programa pontua o propósito inicial do PAS e os eixos com mais força na atuação da PPS no município que ela atua, revelando também, no seu depoimento, o comprometimento com os eixos prioritários de atuação do programa que vão em direção à promoção de saúde para além das atividades físicas/práticas corporais:

O programa surge em 2011, como mais uma estratégia no enfrentamento das doenças crônicas não transmissíveis (DCNT) [...] além do tratamento medicamentoso que o usuário tanto quer, o tratamento das DCNT tem que ir para outros lados, alimentação saudável, que está contemplada dentro do eixo de atuação da Academia da Saúde, além da atividade física, das práticas corporais. (C7, entrevista).

A orientação geral para a implementação do programa, como mostra a entrevistada, indica que as atividades físicas e práticas corporais faziam parte de um conjunto mais amplo de ações que necessitavam ser articuladas para a promoção da saúde da população, uma abordagem que, segundo os profissionais entrevistados, esteve pouco presente na formação inicial em Educação Física. Muitos PEFS relataram pouca ou quase nenhuma abordagem em relação aos campos da promoção de saúde e das políticas públicas ao longo da graduação, como revelam os relatos a seguir:

O curso de EF é voltado pra se trabalhar de forma privada, não de forma pública [...] não há um equilíbrio, o que seria a EF do setor privado, e uma EF do setor público, tanto no âmbito do lazer, esporte e saúde. (P1, entrevista). 
A saúde, eu acho que na verdade, uma cadeira de Saúde Coletiva, acho muito superficial na nossa graduação [...] seis meses eu acho muito pouco pra tu aprofundar no SUS, dentro da área da saúde. (P4, entrevista).

Não tivemos quase nada, uma pincelada só sobre a coisa, eu acho que até o SUS quando eu me formei, não era assim tão grande do jeito que tá. (P6, entrevista).

Eu não sei como está o currículo agora, mas na época a gente não teve nada relacionado a SUS e essas questões. (P5, entrevista).

Os PEFS concluíram a graduação em diferentes instituições e em diferentes momentos históricos. Sobre isso, sabemos que a partir dos anos 2000, com a implementação de políticas como o Programa Nacional de Reorientação da Formação Profissional em Saúde, PRÓ-Saúde I (BRASIL, 2005), e PRÓ-Saúde II (BRASIL, 2007), os cursos do campo da Saúde passaram a reformular seus currículos para incluir temáticas relacionadas ao SUS. Porém, é preciso destacar que algumas instituições foram além das exigências mínimas e estruturaram de forma mais consistente o conteúdo em seus currículos, como foi no caso da experiência do colaborador 2:

$\mathrm{Na}$ minha graduação, tive várias disciplinas que abordaram o SUS, Corporeidade 3 é Educação Física e Saúde Coletiva, Educação Física e SUS, é no terceiro semestre, tem uma carga horária bem grande, semanal, a ideia é propor intervenções no SUS [...] no $5^{\circ}$ semestre tem a disciplina de Saúde Coletiva que é eletiva, mas está em vias de ser obrigatória [...] tem a disciplina de Estudos da Saúde que é obrigatória. [...] Tu enxergas Políticas Públicas de Saúde para Educação Física. Como se insere, lugares possíveis, mais voltadas para o SUS, discute qualidade de vida, quais as possibilidades de formação e a bagagem teórica para isso. (P2, entrevista).

De modo geral, o trabalho de campo revelou que a inserção de PEFS nas PPS é crescente e sua atuação tende a transcender a prática focada nas atividades específicas da formação para incluir aquelas relacionadas ao trabalho interdisciplinar, ainda que algumas PPS favoreçam isso mais do que outras. Conforme nossos interlocutores, há uma fragilidade na formação em EF que vem sendo minimizada com a interação entre os núcleos disciplinares e os espaços de educação permanente nas próprias equipes, no cotidiano de trabalho. Na nossa próxima categoria de análise, se debaterá como o trabalho em equipe tem desempenhado um papel importante diante dos déficits apontados nesta seção.

\subsection{TRABALHO EM EQUIPE E DIA A DIA DAS PPS: “JUNTO E MISTURADO”}

O trabalho em equipe das PPS investigadas acontecia de forma distinta nas três PPS dos municípios pesquisados. Observamos que a busca por se integrar e trabalhar em equipe se dava pela aproximação e inserção dos PEFS no campo, porém, as práticas de núcleo tinham maior protagonismo em momentos e PPS diferentes. Por exemplo, no PAS grande parte das práticas eram voltadas ao núcleo da EF, enquanto no NASF-AB percebeu-se uma mescla de atividades do núcleo da EF e do campo que auxiliavam na articulação com as demais atividades da PPS. Já nas PICs, o trabalho de campo revelou um movimento que transcendia a questão de núcleo, evocando outros saberes, de modo que os relatos sinalizaram 
a necessidade de diálogo entre os diferentes núcleos profissionais na construção conjunta do cuidado com a população usuária das PPS, como ilustra a C7:

A equipe multiprofissional não é só porque está na política pública, ela deve existir na prática, assim a gente está junto e misturado! [...] Um aprende com o outro. Quem tem a ganhar com isso? Não só a gente, mas o usuário! (C7, entrevista).

A oportunidade de se colocar em um grupo com outros profissionais, com outras formações, com outros conhecimentos sobre saúde e doença, foi um momento de grande aprendizado! (P1, entrevista)

Eu me vejo antes como um profissional da Saúde, um sanitarista, aí para as questões de núcleo, sim Educação Física, que é o aporte que vou operar com a Saúde Coletiva. (P2, entrevista)

Essas falas dão o tom de como são os processos de trabalho na composição das equipes, em que os afazeres se misturavam e as linhas que dividiam os núcleos ficavam borradas, da mesma forma que os achados de outros estudos (PEDUZZI, 2001; FERREIRA et al., 2017). Esse funcionamento parecia se reproduzir para além dos profissionais também nas relações no dia a dia, na forma de integração da equipe, nos processos de trabalho e nas decisões a serem tomadas quanto às ações das PPS. Tais aspectos definem se o coletivo de trabalhadores está realmente integrado ou se é apenas um agrupamento de sujeitos de distintos núcleos. Desta forma, foi possível perceber, na pesquisa, elementos que indicam o funcionamento de equipes integradas, em que o protagonismo era circulante entre os núcleos profissionais, de acordo com cada ação a ser desenvolvida.

Tal integração sugere que "estar junto e misturado" é avançar junto nos processos cotidianos de aprendizado e de Educação Permanente. Como revela C7 em conversa informal durante a primeira aproximação, os estudantes de EF chegam muito "crus e saem prontos para o trabalho em saúde" (Diário de Campo), mostrando a importância dos processos educativos e coletivos para a produção de cuidado em saúde a partir da "mistura" com a equipe multiprofissional em Saúde.

Tais achados corroboraram o que outros estudos apontaram em relação a um novo movimento crítico para a inserção da EF no campo da Saúde (LUZ, 2007; DAMICO; KNUTH, 2014; CARVALHO, 2016), avançando desde ações somente prescritivas para a construção de práticas mais conectadas com as realidades de cada território e com a cultura, centrada na população usuária e suas reais necessidades em saúde. Verificou-se uma busca por composição de saberes e não apenas voltada à tradição biologicista, hegemônica no núcleo da EF.

Assim, a disputa epistemológica entre os paradigmas biomédico e sociocultural, presente nos debates acadêmicos, não se reproduziu no campo da pesquisa uma vez que os relatos da população usuária das PPS indicavam que existia mais consensos do que dissensos, estando ambas as perspectivas conectadas no dia a dia das PPS, ao considerarmos sua experiência de cuidado em saúde nos territórios investigados.

\subsection{A CLÍNICA DA EDUCAÇÃO FÍSICA: UMA CLÍNICA MUTANTE}

Essa categoria discute algumas pistas sobre a práxis de PEFS, bem como tenta reunir subsídios para pensar uma possível Clínica da Educação Física. De 
modo geral, as análises revelam que a construção da Clínica da Educação Física requer dos PEFS a compreensão das demandas e propósitos de cada PPS, para, assim, adequar as ferramentas do núcleo na composição com o campo, de modo situacional.

Wachs (2017) delineia uma possível Clínica da Educação Física, apontando uma discussão sobre o cuidado em saúde produzido por PEFS, por alguns vieses e dilemas entre a função pedagógica ou sanitária, que causa dúvidas, tanto para gestores quanto profissionais, para a inserção do núcleo no campo da Saúde. Existem questões pedagógicas nas funções sanitárias de PEFS nas PPS, assim como há muito a ser explorado nas práticas de educação em saúde, o que demonstra o quanto as funções pedagógica e sanitária estão juntas nos processos de educação permanente das equipes e nas ações de educação em saúde das PPS.

Percebemos que cada política pesquisada tinha um nível de inserção dos PEFS e, consequentemente, o desenvolvimento de ações voltadas ao núcleo da EF. No PAS, as ferramentas do núcleo da EF eram mais evocadas, de modo que os elementos da cultura corporal se constituíam em "carro-chefe" da política. Contudo, a ênfase na cultura corporal partia da compreensão da necessidade do vínculo com a população usuária e seu território, da mobilização comunitária, das relações com a rede intersetorial de políticas públicas e da sensibilidade cultural. Tais aspectos, concernentes ao campo da saúde, como integralidade do cuidado, acolhimento e escuta do usuário, assumiam centralidade neste processo dinâmico, levando em conta as dimensões biopsicossociais.

Já no NASF-AB, o foco de atuação dos profissionais era o matriciamento, definido como o apoio técnico-assistencial às equipes de saúde (CAMPOS; DOMITTI, 2007). Nesse contexto, os conhecimentos sobre práticas pedagógicas - de educação continuada e permanente - a serem desenvolvidas com as equipes assistidas e a composição da atenção em conjunto com a população usuária mostraram-se essenciais para os PEFS (OLIVEIRA; WACHS, 2018). Assim, as ferramentas do núcleo eram acionadas a partir de contextos específicos de manejo de casos ou de apoio à formulação de ações de prevenção e promoção da saúde comunitária. Encontramos no NASF-AB exatamente esta mescla de práticas, ou seja, tanto atividades de núcleo - como grupos de ginástica, atendimentos individuais para reabilitação física, consultoria para grupos de caminhada - como aquelas ligadas ao campo - como matriciamento, reuniões de equipe com os diversos níveis de atenção (atenção básica, serviços especializados e hospital municipal), tutoria a residentes e estudantes, entre outras atividades e demandas que emergiam da práxis cotidiana.

Particularmente nas PICS, os conhecimentos e práticas acionados - na sua grande maioria inspirados nas culturas orientais - requerem formações não disponíveis nas grades curriculares da formação em EF ou mesmo de outras profissões do campo da saúde. Mesmo assim, busca-se estabelecer um diálogo dos conhecimentos da cultura corporal com os oriundos da anatomia e fisiologia, mesmo que inseridos no âmbito de outra racionalidade, como ilustra $\mathrm{P} 1$ :

Hoje a gente fala de loga e Danças Circulares, das práticas corporais da tradição chinesa, de meditação, que se aproxima da EF. Acaba que estar 
atuando nas PICs e ser da EF me dá certa liberdade. [...] Contempla as questões da EF e do corpo. Mas numa perspectiva que nenhuma PICs pertence ao núcleo profissional da EF, mas elas também podem ser realizadas por este profissional. ( $\mathrm{P} 1$, entrevista).

A fala de P1 vai ao encontro daquilo que outros estudos discutem a respeito da inclusão das PICs dos serviços de saúde, destacando a interdisciplinaridade de tais práticas e a diversidade dos núcleos profissionais que as ofertam (visto que são atividades pouco desenvolvidas de modo específico nas graduações da área da saúde). Ainda se visualiza a necessidade de se trabalhar com as PICs de modo a ampliar as concepções de corpo e processo saúde-doença para além das perspectivas mais tradicionais da racionalidade biomédica (ANTUNES et al., 2018).

Diante disso, em consonância com outros trabalhos que discutem a práxis da EF nas PPS (AMARAL JÚNIOR, 2017; CARVALHO, 2005; DAMICO, 2020; FERREIRA, 2013; PIRES, 2014), os dados produzidos pela pesquisa nos permitem delinear uma noção de que a Clínica da Educação Física é mutante e constituída por muitas clínicas, na articulação entre PPS, territórios e equipes. Mas, para que isso aconteça de modo efetivo, é fundamental sanar o déficit existente na formação de PEFS para a inserção no campo da Saúde, bem como ampliar a oferta de postos de trabalho nas PPS, o que consolidaria mudanças e permitiria ampliar a promoção de saúde da população em conjunto com os demais núcleos.

\section{CONSIDERAÇÕES FINAIS}

No presente estudo buscamos analisar e entender os processos relativos ao trabalho de PEFS nas PPS na Região Metropolitana de Porto Alegre (Programa Academia da Saúde, o Núcleo Ampliado de Saúde da Família e Atenção Básica e Política de Práticas Integrativas e Complementares em Saúde), bem como compreender os percursos formativos desses sujeitos. Foi possível identificar os diferentes elementos que compõem o cotidiano de trabalho dos PEFS nesses espaços de atuação e tecer algumas análises e reflexões acerca de temáticas como a influência da formação inicial, o trabalho em equipe multiprofissional e os referenciais que ajudam a constituir os modos de fazer a clínica na Educação Física.

O trabalho de campo junto aos PEFS nos revela que, apesar da pouca tradição do núcleo da EF no campo da saúde coletiva e nas equipes dos serviços públicos de saúde, o ingresso desses trabalhadores nas PPS tem se mostrado crescente e que sua atuação, muitas vezes, extrapola a prescrição e orientação de atividades físicas, adquirindo um caráter mais próximo da interdisciplinaridade (embora isso não ocorresse da mesma forma nos serviços pesquisados).

A referência ao trabalho interdisciplinar também diz respeito à organização e dinâmica das equipes do PAS, NASF-AB e PICS. Foi possível identificar que o protagonismo das ações de cuidado variava entre os distintos núcleos profissionais, sendo o vínculo com os usuários, com o território e o tipo de intervenção terapêutica a ser mobilizada, os critérios mais utilizados para determinar a coordenação das atividades - podendo inclusive ser realizadas por mais de um núcleo. 
Os achados nos permitem delinear uma noção de que a Clínica da Educação Física se faz mutante, de maneira artesanal, sendo constituída por referências das ciências biológicas e das ciências humanas e sociais e pelos saberes populares e culturais, buscando manter uma articulação entre políticas públicas, comunidades e equipes. Para que isso ocorra de modo mais frequente e qualificado, se faz necessária a ampliação da oferta de postos de trabalho nessas políticas, de modo que seja viável uma inserção sólida e em condições materiais, subjetivas e econômicas (carreira, salário e gestão) que potencializem o trabalho no campo da Saúde junto com os demais núcleos profissionais.

De acordo com nossos interlocutores, há uma fragilidade na formação em EF que vem sendo minimizada com a interação entre os núcleos disciplinares e os espaços de educação permanente nas próprias equipes, no cotidiano de trabalho. Portanto, entendemos que a formação inicial necessita ainda de mais espaços que oportunizem a vivência e a experiência dos estudantes nas PPS, assim como atividades que os auxiliem a pensar sobre as diferentes clínicas da EF. Acreditamos que modelos plurais de clínica podem vir a tornar-se ferramentas potentes para a constituição dos processos de trabalho que envolvem a Educação Física, seja nas práticas de proteção, recuperação e promoção de saúde.

Por fim, compreendemos os limites de nossa pesquisa, por contemplar poucos territórios. Reafirmamos a necessidade da realização de novas investigações que possam pesquisar outras realidades, serviços e equipes de saúde, de modo a contribuir para uma maior compreensão das possibilidades de atuação dos PEFS no campo das políticas públicas. E assim se possa cada vez mais integrar-se com os demais núcleos para o fortalecimento das práticas de promoção de saúde e a efetivação do princípio da integralidade.

\section{REFERÊNCIAS}

AMARAL JÚNIOR, Alpheu Ferreira do. Vestígios do processo de medicalização da vida na atenção básica. 2017. 138 f. Dissertação (Mestrado em Saúde Coletiva) - Escola de Enfermagem, Universidade Federal do Rio Grande do Sul, Porto Alegre, 2017.

ANTUNES, Priscilla de Cesaro et al. Revisão sistemática sobre práticas corporais na perspectiva das práticas integrativas e complementares em saúde. Motrivivência, v. 30, n. 55, p. 227-247, set. 2018.

BILIBIO, Luiz Fernando; DAMICO, José Geraldo Soares. Carta a um jovem professor. Cadernos de Formação RBCE, v. 2, n. 2, p. 92-103, 2011.

BRASIL. Portaria n. 256, de 11 de março de 2013. Estabelece novas regras para o cadastramento das equipes que farão parte dos Núcleos de Apoio à Saúde da Família (NASF) Sistema de Cadastro Nacional de Estabelecimentos de Saúde (SCNES). Diário Oficial da República Federativa do Brasil, Brasília, DF, n. 50, p. 43, $2013 a$.

BRASIL. Portaria n. 2.681 MS/GM, de 7 de novembro de 2013. Redefine o Programa Academia da Saúde no âmbito do Sistema Único de Saúde (SUS). Diário Oficial da República Federativa do Brasil, Brasília, DF, n. 218, p. 37-38, 2013b. 
BRASIL. Portaria $n^{\circ} 3.588 \mathrm{GM} / \mathrm{MS}$, de 21 de dezembro de 2017. Altera as Portarias de Consolidação n. 3 e n. 6, para dispor sobre a Rede de Atenção Psicossocial e dá outras providências. Diário Oficial da República Federativa do Brasil, Brasília, DF, n. 245, p. 236-238, 2017a.

BRASIL. Portaria n. 2.436 GM/MS, de 21 de setembro de 2017. Aprova a Política Nacional de Atenção Básica, estabelecendo a revisão de diretrizes para a organização da Atenção Básica, no âmbito do Sistema Único de Saúde (SUS). Diário Oficial da República Federativa do Brasil, Brasília, DF, n. 183, p. 68, 2017b.

BRASIL. Portaria interministerial $n^{\circ} 2.101$, de 3 de novembro de 2005. Institui o Programa Nacional de Reorientação da Formação Profissional em Saúde - Pró-Saúde - para os cursos de graduação em Medicina, Enfermagem e Odontologia. Diário Oficial da República Federativa do Brasil, Brasília, DF, n. 212, p. 111, 2005.

BRASIL. Portaria interministerial n. 3.019, de 26 de novembro de 2007. Institui o Programa Nacional de Reorientação da Formação Profissional em Saúde - Pró-saúde II. Diário Oficial da República Federativa do Brasil, Brasília, DF, n. 227, p. 44, 2007.

BRAUN, Virginia; CLARKE, Victoria. Using thematic analysis in psychology. Qualitative Research in Psychology, v. 3, n. 2, p. 77-101, 2006. Disponível em: https://www.tandfonline. com/doi/abs/10.1191/1478088706qp063oa. Acesso em: 14 de nov. 2020.

CAMPOS, Gastão Wagner de Sousa. Saúde pública e saúde coletiva: campo e núcleo de saberes e práticas. Sociedade e Cultura, v. 3, n. 1-2, p. 51-74, 2000.

CAMPOS, Gastão Wagner de Sousa et al. (org.). Tratado de Saúde Coletiva. 2. ed. São Paulo: Hucitec, 2012.

CAMPOS, Gastão Wagner de Sousa; DOMITTI, Ana Carla. Apoio matricial e equipe de referência: uma metodologia para gestão do trabalho interdisciplinar em saúde. Cadernos de Saúde Pública, v. 23, n. 2, p. 399-407, fev. 2007.

CARVALHO, Fábio Fortunato Brasil de. Práticas corporais e atividades físicas na Atenção Básica do Sistema Único de Saúde: ir além da prevenção das doenças crônicas não transmissíveis é necessário. Movimento (Porto Alegre), v. 22, n. 2, p. 647-658, abr./jun. 2016. DOI: https://doi.org/10.22456/1982-8918.58174

CARVALHO, Yara Maria. Entre o biológico e o social: tensões no debate teórico acerca da saúde na educação física. Motrivivência, v. 17, n. 24, p. 97-105, 2005.

DAMICO, José Geraldo Soares. A educação física menor na saúde coletiva. In: WACHS, Felipe; LARA, Larissa; ATHAYDE, Pedro (org.). Atividade física e saúde: ciências do esporte, educação física e produção do conhecimento em 40 anos de CBCE. Natal: EDUFRN, 2020. p. 29-50.

DAMICO, José Geraldo Soares. Das possibilidades às incertezas: instrumentos para intervenção profissional de educação física no posto de saúde. In: FRAGA, Alex Branco; WACHS, Felipe (org.). Educação Física e Saúde Coletiva: políticas de formação e perspectivas de intervenção. Porto Alegre: UFRGS, 2007. p. 73-86.

DAMICO, José Geraldo Soares; KNUTH, Alan Goularte. O des(encontro) das práticas corporais e atividade física: Hibridizações e borramentos no campo da saúde. Movimento (Porto Alegre), v. 20, n. 1, p. 329-350, jan/mar. 2014. DOI: https://doi.org/10.22456/1982$\underline{8918.39474}$ 
FERREIRA, Luiz Alberto dos Santos. 0 trabalho da educação física na composição de equipe de saúde mental especializada em álcool e outras drogas. 2013. $108 \mathrm{f}$. Dissertação (Mestrado em Ciências do Movimento Humano) - Escola de Educação Física, Fisioterapia e Dança, Universidade Federal do Rio Grande do Sul, Porto Alegre, 2013.

FERREIRA, Luiz Alberto dos Santos et al. Entre a composição e a tarefa: estudo de caso sobre a inserção da educação física em um serviço de saúde mental. Revista Brasileira de Ciências do Esporte, v. 39, n. 2, p. 176-182, 2017.

FURTADO, Roberto Pereira. et al. Educação Física e saúde mental: uma análise da rotina de trabalho dos profissionais dos CAPS de Goiânia. Movimento (Porto Alegre), v. 22, n. 4 , p. 1077-1090, 2016. DOI: https://doi.org/10.22456/1982-8918.62158

HALLAL, Pedro Curi; MELO, Victor Andrade de. Crescendo e enfraquecendo: um olhar sobre os rumos da Educação Física no Brasil. Revista Brasileira de Ciências do Esporte, v. 39, n. 3, p. 322-327, 2017.

LUZ, Madel Therezinha. Educação Física e saúde coletiva: papel estratégico da área e possibilidades quanto ao ensino na graduação e integração na rede de serviços públicos de saúde. In: FRAGA, Alex Branco; WACHS, Felipe (org.). Educação Física e Saúde Coletiva: políticas de formação e perspectivas de intervenção. Porto Alegre: UFRGS, 2007. p. 9-16.

MACHADO, Carlos Eduardo. Práxis: trabalho e ideia prática em Hegel. Filosofia e Educação, v. 10, n. 2, p. 438-451, maio/ago. 2018.

MARX, Karl; ENGELS, Friedrich. A Ideologia Alemã. São Paulo: Boitempo, 2007.

NOGUEIRA, Júlia Aparecida Devidé; BOSI, Maria Lúcia Magalhães. Saúde Coletiva e Educação Física: distanciamentos e interfaces. Ciência \& Saúde Coletiva, v. 22, n. 6, p. 1913-1922, 2017.

OLIVEIRA, Bráulio Nogueira de; WACHS, Felipe. Educação Física e atenção primária à saúde: apropriações acerca do apoio matricial. Movimento (Porto Alegre), v. 24, n. 1, p. 173-186, jan./mar. 2018. DOI: https://doi.org/10.22456/1982-8918.69965

PAIM, Jairnilson Silva; ALMEIDA-FILHO, Naomar. Saúde coletiva: uma "nova saúde pública" ou campo aberto a novos paradigmas? Revista Saúde Pública, v. 32, n. 4, p. 299-316, 1998.

PEDUZZI. Marina. Equipe multiprofissional em Saúde: conceitos e tipologia. Revista Saúde Pública, v. 35, n. 1, p. 103-109, 2001.

PIRES, Cássio Lamas. Educação Física e Saúde Coletiva: flexões, inflexões e outras interferências constantes. 2014. 142 f. Dissertação (Mestrado em Saúde Coletiva) - Escola de Enfermagem, Universidade Federal do Rio Grande do Sul, Porto Alegre, 2014.

SANTOS, Deise Francelle dos. Educação Física no Hospital Psiquiátrico São Pedro: uma experiência de familiarização e estranhamento nas entrelinhas dos relatos de estágio. 2016. 119 f. Dissertação (Mestrado em Ciências do Movimento Humano) - Escola de Educação Física, Fisioterapia e Dança, Universidade Federal do Rio Grande do Sul, Porto Alegre, 2016.

SILVA, Renatho Andriolla da Silva. O conceito de práxis em Marx. 2017. 104 f. Dissertação (Mestrado em Filosofia) - Centro de Ciências Humanas, Letras e Artes, Universidade Federal do Rio Grande do Norte, Natal, 2017. 
SOUZA, Luciana Karine de. Pesquisa com análise qualitativa de dados: conhecendo a Análise Temática. Arquivos Brasileiros de Psicologia, v. 71, n. 2, p. 51-67, 2019.

TURATO, Egberto Ribeiro. Tratado da metodologia da pesquisa clínico-qualitativa. Petrópolis: Vozes, 2003.

VAZ, Fabiana Fernandes. Análise da distribuição dos profissionais de Educação Física nos serviços de saúde do Estado do Rio Grande do Sul. 2014. 38 f. Trabalho de Conclusão de Curso (Graduação em Educação Física Bacharelado) - Escola de Educação Física, Fisioterapia e Dança, Universidade Federal do Rio Grande do Sul, Porto Alegre, 2014.

WACHS, Felipe. Educação Física e o Cuidado em Saúde: explorando alguns vieses de aprofundamento para a relação. Saúde em Redes, v. 3, n. 4, p. 339-349, 2017. 


\section{(10 MOVIMENTO

Abstract: This study sought to discuss the insertion of Physical Education Professionals (PEFS) in Public Health Policies (PPS), analyzing the tensions between the nucleus and the field from their entry into policies such as the Family Health Support Center, Health Academy Program and Complementary Integrative Practices in Health team. To produce data, visits to health services, in-depth interviews, observations and notes in a field diary were carried out, using Thematic Analysis of the empirical material. The study pointed out that there are similarities and differences in the practices of professionals working in PPS that transcend the Physical Education nucleus, always seeking a closer relationship with the field of Health. The findings revealed the potential of building a Physical Education Clinic that relates to the health needs of the user population.

Keywords: Physical Education. Public Health. Delivery of Health Care.

Resumen: Este estudio ha buscado discutir la inserción de Profesionales de Educación Física (PEFS) en Políticas Públicas de Salud (PPS) y analizar las relaciones entre núcleo y campo. Para producir los datos se realizaron visitas al Núcleo de Apoyo a la Salud de la Familia (NASF), al Programa Academia de la Salud (PAS) y al equipo de Prácticas Integrativas Complementarias en Salud (PICs). También realizamos entrevistas en profundidad, observaciones y anotaciones en un diario de campo, utilizando Análisis Temático del material empírico. El estudio mostró que existen semejanzas y diferencias en las prácticas de los profesionales que actúan en las PPS que trascienden el núcleo de la Educación Física y buscan siempre una mayor aproximación con el campo de la salud, lo que revela el potencial para la construcción de una Clínica de la Educación Física que esté conectada con las necesidades de salud de la población usuaria.

Palabras clave: Educación Física. Salud Pública. Atención a la Salud. 


\section{(10 MOVIMENTO

NOTAS

\section{LICENÇA DE USO}

Este é um artigo publicado em acesso aberto (Open Access) sob a licença Creative Commons Atribuição 4.0 Internacional (CC BY 4.0), que permite uso, distribuição e reprodução em qualquer meio, desde que o trabalho original seja corretamente citado. Mais informações em: https://creativecommons.org/licenses/by/4.0

\section{CONFLITO DE INTERESSES}

Os autores declararam que não existe nenhum conflito de interesses neste trabalho.

\section{CONTRIBUIÇÕES AUTORAIS}

Luiz Alberto dos Santos Ferreira: Conceituação, Análise Formal, Investigação, Metodologia, Escrita e Revisão.

Tonantzin Ribeiro Gonçalves: Análise Formal, Metodologia, Supervisão, Orientação, Escrita e Revisão.

Leonardo Trápaga Abib: Análise Formal, Escrita e Revisão.

\section{FINANCIAMENTO}

Não houve financiamento por órgãos de pesquisa.

\section{ÉTICA DE PESQUISA}

O presente trabalho apresentado ao Comitê de Ética da UNISINOS, projeto n. 4.671. 150 e todos os procedimentos éticos que constam das Resoluções 466/12 e 510/ 2016 do Conselho Nacional de Saúde do Brasil foram devidamente contemplados.

\section{COMO REFERENCIAR}

FERREIRA, Luiz Alberto dos Santos; GONÇALVES, Tonantzin Ribeiro; ABIB, Leonardo Trápaga. A clínica da educação física nas políticas públicas de saúde: interfaces entre núcleo e campo. Movimento, v.28, p. e28002, jan./dez. 2022. DOI: https://doi.org/10.22456/1982-8918.116321. Disponível em: https://seer.ufrgs. br/Movimento/article/view/116321. Acesso em: [dia] [mês abreviado]. [ano].

\section{RESPONSABILIDADE EDITORIAL}

Alex Branco Fraga*, Elisandro Schultz Wittizorecki*, Ivone Job*, Mauro Myskiw*, Raquel da Silveira*

*Universidade Federal do Rio Grande do Sul, Escola de Educação Física, Fisioterapia e Dança, Porto Alegre, RS, Brasil. 